\title{
On asymptotic stability of continuous-time risk-sensitive filters with respect to initial conditions
}

\author{
Subhrakanti Dey ${ }^{\mathrm{a}, *}$, Charalambos D. Charalambous ${ }^{\mathrm{b}}$ \\ ${ }^{a}$ Department of Electrical \& Electronic Engineering, University of Melbourne, Parkville, Victoria 3052, Australia \\ ${ }^{\mathrm{b}}$ Department of Electrical Engineering, McGill University, Montreal, Canada H3A 2 A7
}

Received 4 August 1998; accepted 28 March 2000

\begin{abstract}
In this paper, we consider the problem of risk-sensitive filtering for continuous-time stochastic linear Gaussian time-invariant systems. In particular, we address the problem of forgetting of initial conditions. Our results show that suboptimal risk-sensitive filters initialized with arbitrary Gaussian initial conditions asymptotically approach the optimal risk-sensitive filter for a linear Gaussian system with Gaussian but unknown initial conditions in the mean square sense at an exponential rate, provided the arbitrary initial covariance matrix results in a stabilizing solution of the $\left(H_{\infty}\right.$-like) Riccati equation associated with the risk-sensitive problem. More importantly, in the case of non-Gaussian initial conditions, a suboptimal risk-sensitive filter asymptotically approaches the optimal risk-sensitive filter in the mean square sense under a boundedness condition satisfied by the fourth order absolute moment of the initial non-Gaussian density and a slow growth condition satisfied by a certain Radon-Nikodym derivative. (c) 2000 Elsevier Science B.V. All rights reserved.
\end{abstract}

Keywords: Risk-sensitive filtering; Riccati equation; Forgetting of initial conditions; Non-Gaussian density; Mean square convergence

\section{Introduction}

Risk-sensitive filtering optimizes an exponential of quadratic (or more general convex) cost criterion. As opposed to $L_{2}$ filtering, risk-sensitive filtering penalizes the higher order moments of the estimation error energy, thus making the filters useful in uncertain plant and noise environments. It also allows a trade-off between optimal filtering for the nominal model case and the average noise situation, and robustness to worst case noise and model uncertainty by weighting the index of the exponential by a risk-sensitive parameter. For example, it has been shown in [12] that discrete-time risk-sensitive filters for hidden Markov

\footnotetext{
* Corresponding author.
}

models (HMM) with finite-discrete states perform better than standard HMM filters in situations involving uncertainties in the noise statistics. A more recent work [6] shows that such risk-sensitive filters enjoy an error bound which is the sum of two terms, the first of which coincides with an upper bound on the error one would obtain if one knew exactly the underlying probability model, while the second term is a measure of the distance between the true and design probability models. Although risk-sensitive filtering was introduced for discrete-time linear systems in [24], the term "risk-sensitive filtering" was introduced in [13] and more general discrete-time nonlinear systems were treated, using similar techniques of [17] in the context of risk-sensitive control. Apart from the potential usefulness of risk-sensitive filters 
in uncertain environments, risk-sensitive problems, in the small noise-limit, have been shown to be closely related to estimation/control problems in a deterministic worst-case noise scenario given from a differential game $\left(H_{\infty}\right.$ estimation/control problems for linear systems ) $[25,26,17,19,8]$.

It is well known that the mean of the conditional density of the state given the observations for a stochastic state space-signal model achieves the minimum variance filter. For a linear Gaussian system with known or Gaussian distributed initial conditions, the conditional density is Gaussian and given by its mean and covariance (which can be calculated off-line from a Riccati differential equation). This is also popularly known as a Kalman filter. On the other hand, the optimal estimation problem becomes an essentially non-linear problem if the initial condition is not Gaussian distributed. However, for linear Gaussian systems, it has been shown [18,21] that the optimal filter (or its density) can be given by a finite number of statistics, which constitute the optimal (in the minimum variance sense) filter for an augmented linear system. The initial condition is often not known and it is often unrealistic to assume that the initial condition has a Gaussian density. However, it has been shown in [21] (continuous-time) [23] (discrete-time) that the conditional density filter forgets the initial condition asymptotically at an exponential rate. In other words, one can assume a Gaussian density for the initial condition and use a suboptimal Kalman filter which asymptotically becomes optimal, provided the actual density of the initial condition has finite first- and second-order moments. Exponential stability results for discrete-time filters have been shown in [7] and for Benes filters [3] in [22]. Also, stability results for filters based on Lyapunov exponents have been explored in $[14,1,2]$.

It is also well known that the optimal risk-sensitive filter for a continuous-time linear Gaussian system with a Gaussian initial condition is an $H_{\infty}$ filter $[9,11]$. Analogous results for discrete-time linear signal models can be found in [24,13]. In the case of a non-Gaussian initial condition, the risk-sensitive estimation problem, as can be expected, becomes a nonlinear problem in general.

In this paper, our objective is to address the problem of risk-sensitive estimation for non-Gaussian initial conditions for continuous-time linear Gaussian time-invariant systems. We also study the asymptotic behaviour of the optimal risk-sensitive estimate with respect to its initial conditions. This study is done in two parts. First, we study the asymptotic effects of arbitrary Gaussian initial conditions on the optimal risk-sensitive estimate (i.e., different values of the initial covariance, the mean is taken to be zero without loss of generality) for linear Gaussian systems with Gaussian initial conditions. The second part studies the asymptotic effects of a non-Gaussian initial condition, and the behaviour of the distance of the optimal risk-sensitive filter from the suboptimal risk-sensitive filter (with a Gaussian initial condition assumption). Our results show that we get mean square convergence asymptotically in both the above studies under certain conditions. In other words, one can compute a suboptimal risk-sensitive filter with arbitrary Gaussian initial conditions (provided the initial covariance results in a stabilizing solution for the $\left(H_{\infty}\right.$-like) algebraic Riccati equation associated with the risk-sensitive problem) and this filter will asymptotically converge to the optimal risk-sensitive filter in the mean square sense for unknown Gaussian initial conditions and unknown non-Gaussian initial conditions satisfying certain constraints.

In Section 2, we introduce the signal model, the risk-sensitive estimation problem and reformulate it under a new probability measure. In Section 3, we briefly present the optimal risk-sensitive filter for linear Gaussian systems with Gaussian initial conditions and show the asymptotic stability of these filters with respect to arbitrary Gaussian initial conditions. Section 4 deals with non-Gaussian initial conditions where we first derive the optimal risk-sensitive filter using the information state approach and then we show the asymptotic mean square convergence properties of such filters with respect to their initial conditions. Section 5 presents some concluding remarks.

\section{Signal model}

Consider a complete probability space $(\Omega, \mathscr{F}, \mathscr{P})$ on which we define the following stochastic linear time-invariant state space model:

$$
\begin{aligned}
& \mathrm{d} x_{t}=F x_{t} \mathrm{~d} t+G \mathrm{~d} w_{t}, \quad x_{0} \sim \Pi_{0}\left(x_{0}\right), \\
& \mathrm{d} y_{t}=H x_{t} \mathrm{~d} t+\mathrm{d} v_{t}, \quad y_{0}=0 .
\end{aligned}
$$

Here, $x_{t} \in \mathbb{R}^{n}, y_{t} \in \mathbb{R}^{p}, x_{0} \in \mathbb{R}^{n}$. The process noise $w_{t} \in \mathbb{R}^{n}$ and the measurement noise $v_{t} \in \mathbb{R}^{p}$ are Wiener processes with covariance $I_{n}$ and $I_{p}$, respectively. Also, $G G^{*}=\Sigma_{w} \geqslant 0 . \Pi_{0}$ is not necessarily Gaussian. 
We assume that $x_{0}, w_{t}, v_{t}$ are mutually independent and that $(F, G)$ are stabilizable and $(F, H)$ are detectable.

Denote the complete filtration generated by the observation $\sigma$-algebra, namely, $\sigma\left\{y_{s}, 0 \leqslant s \leqslant t\right\}$ as $\left\{\mathscr{Y}_{t}\right\}$, the complete filtration generated by $\sigma\left\{x_{0}\right\} \vee \sigma\left\{w_{s}, 0 \leqslant s \leqslant t\right\}$ as $\left\{\mathscr{F}_{t}\right\}$ and the complete filtration generated by $\sigma\left\{y_{s}, 0 \leqslant s \leqslant t\right\} \vee \sigma\left\{x_{0}\right\} \vee \sigma\left\{w_{s}\right.$, $0 \leqslant s \leqslant t\}$ as $\left\{\mathscr{G}_{t}\right\}$.

\subsection{Risk-sensitive estimation}

We define the risk-sensitive estimation problem for the continuous-time stochastic linear system (1) as to obtain a $\mathscr{Y}_{t}$-measurable stochastic process $\hat{x}_{t} \in \mathbb{R}^{n}$ (assumed to be well-defined) such that

$$
\begin{aligned}
\hat{x}_{t} \in \underset{\zeta}{\operatorname{argmin}} E\left[\operatorname { e x p } \mu \left\{\int_{0}^{t} l\left(x_{s}, \hat{x}_{s}\right) \mathrm{d} s\right.\right. \\
\left.\left.+\tilde{l}\left(x_{t}, \zeta\right)\right\} \mid \mathscr{Y}_{t}\right] .
\end{aligned}
$$

Here, $E[$.$] denotes expectation under P, \mu>0$ and $l, \tilde{l}: \mathbb{R}^{n} \times \mathbb{R}^{n} \rightarrow \mathbb{R}$ is measurable in $(x, \hat{x})$ and continuous in $\hat{x}$ and is of the following form

$$
\begin{aligned}
& l(x, \hat{x})=\frac{1}{2}(x-\hat{x})^{*}(x-\hat{x}), \\
& \tilde{l}(y, z)=\frac{1}{2}(y-z)^{*}(y-z),
\end{aligned}
$$

where the units of $l(.,$.$) and \tilde{l}(.,$.$) are cost per unit$ time and cost, respectively. At this point, let us include a brief explanation of the risk-sensitive cost (2) as defined above. It should be noted that the above optimization, in the most general setting, assumes that the process $\hat{x}_{s}, s \in[0, t)$ has been obtained and is well-defined, and while optimizing to solve for $\hat{x}_{t}$, essentially we are optimizing at uncountably infinite number of time points. In specific cases however, as we will see later on, one can obtain finite-dimensional representation of the risk-sensitive estimate which is given by a stochastic differential equation (e.g., linear Gaussian systems). Also note that although the term $\exp \mu\left(\int_{0}^{t} l\left(x_{s}, \hat{x}_{s}\right) \mathrm{d} s\right)$ is not affected (because it is a Lebesgue integral) by the value of $\hat{x}_{t}$ (which, in a way, justifies the existence of the terminal cost $\tilde{l}\left(x_{t}, \hat{x}_{t}\right)$ in (2)), it does affect the solution of the optimization problem. In other words, optimizing the cost (2) and optimizing a cost that is defined as

$\hat{x}_{t} \in \underset{\zeta}{\operatorname{argmin}} E\left[\exp \left[\mu \tilde{l}\left(x_{t}, \zeta\right) \mid \mathscr{Y}_{t}\right]\right.$ are completely different optimization problems. In the linear quadratic case (i.e., where the underlying system is linear Gaussian and $l(x, \hat{x})$ is a quadratic function (like (3)), (2) is optimized by an $H_{\infty}$ filter whereas (4) is optimized by a Kalman filter (see [19]). These results have been derived in discrete-time in [24,13] and the justification of having a cost like (2) in relation to the error bounds the risk-sensitive filters achieve and their significance with respect to robustness have been discussed in [6].

Next, we work under a probability measure $\bar{P}$ such that under $\bar{P}, y_{t}$ is Wiener process with covariance $I_{p}$ and independent of $x_{t}$ (and hence $x_{0}$ ). Using Bayes' Theorem, the risk-sensitive estimation problem is re-formulated as

$$
\begin{aligned}
\hat{x}_{t} \in \underset{\zeta}{\operatorname{argmin}} \bar{E}\left[\Lambda _ { t } \operatorname { e x p } \mu \left\{\int_{0}^{t} l\left(x_{s}, \hat{x}_{s}\right) \mathrm{d} s\right.\right. \\
\left.\left.+\tilde{l}\left(x_{t}, \zeta\right)\right\} \mid \mathscr{Y}_{t}\right],
\end{aligned}
$$

where $\Lambda_{t}=\exp \left(\int_{0}^{t}\left(H x_{s}\right)^{*} \mathrm{~d} y_{s}-\frac{1}{2} \int_{0}^{t}\left(H x_{s}\right)^{*}\left(H x_{s}\right) \mathrm{d} s\right)$. For details on this particular application of change of probability measure technique (see [15] (discrete-time) and $[4,11]$ (continuous-time)).

\section{Risk-sensitive estimation with Gaussian initial condition}

In this section, we present the risk-sensitive estimation results for linear Gaussian systems with Gaussian initial conditions and study the asymptotic forgetting property of the estimates with respect to initial conditions. Without loss of generality (see [18]), we take the mean of the initial density to be zero. It is with respect to the covariance matrix of the initial state that we study the asymptotic convergence properties.

The following theorem summarizes the risksensitive estimation results for the linear Gaussian systems with Gaussian initial condition (for similar proofs, see $[11,10])$.

Theorem 3.1. Consider the signal model (1) and the risk-sensitive cost given by (2) and (3). Suppose $x_{0} \sim \mathrm{N}(0, \Sigma)$. The optimal risk-sensitive estimate $\hat{x}_{t}^{G}$ is then given by the following stochastic differential equation (SDE):

$\mathrm{d} \hat{x}_{t}^{G}=F \hat{x}_{t}^{G}+Q_{t} H^{*}\left(\mathrm{~d} y_{t}-H \hat{x}_{t}^{G} \mathrm{~d} t\right), \quad \hat{x}_{0}^{G}=0$, 
where $Q_{t}$ satisfies the following Riccati differential equation (RDE):

$\dot{Q}_{t}=F Q_{t}+Q_{t} F^{*}+\Sigma_{w}-Q_{t}\left(H^{*} H-\mu I\right) Q_{t}, Q_{0}=\Sigma$.

Remark 3.1. Note that the above equations resemble those of the continuous-time $H_{\infty}$ filter, the obvious difference with the Kalman filter lying in the Riccati differential equation (7) due to the presence of the quadratic term with the opposite sign. Much of the asymptotic stability results that are about to follow are hinged on the stability results of the above $H_{\infty}$ Riccati differential equation.

\subsection{Asymptotic optimality of risk-sensitive filters with arbitrary Gaussian initial conditions}

Before proceeding to the case of non-Gaussian initial conditions, we would like to show that the risk-sensitive filters forget the initial conditions when the initial conditions are Gaussian. In other words, we would like to show that no matter which value of the covariance matrix for the initial state we start with (provided it results in a stabilizing solution of the RDE (7)), the risk-sensitive estimate asymptotically approaches the optimal risk-sensitive estimate (the one initialized with the true initial covariance) in the mean square sense. In the case of minimum variance filtering, similar results with mean square and almost sure convergence already exist $[21,23]$ (discrete-time), the best reference (continuous-time) being [21]. Our analysis closely follows theirs, however, unlike the case of the Kalman filtering, the estimation error does not become an innovations process under $P$, and this is where our analysis becomes different. We leave the case of non-Gaussian initial conditions until the next section.

Definition 3.1. Define the stochastic process $\beta_{t}$ by the following stochastic differential equation:

$\mathrm{d} \beta_{t}=\left(F-\Sigma_{t} H^{*} H\right) \beta_{t} \mathrm{~d} t+\Sigma_{t} H^{*} \mathrm{~d} y_{t}, \quad \beta_{0}=0$,

where $\Sigma_{t}$ satisfies the Riccati differential equation $\dot{\Sigma}_{t}=F \Sigma_{t}+\Sigma_{t} F^{*}+\Sigma_{w}-\Sigma_{t}\left(H^{*} H-\mu I\right) \Sigma_{t}, \quad \Sigma_{0}=0$.

Remark 3.2. Note that in view of (6) and (7), $\beta_{t}$ is a suboptimal risk-sensitive estimate where $\Sigma_{t}$ is obtained by solving the same Riccati differential equation, only with a different initial condition.
Remark 3.3. We have not made any statements about the existence of a stabilizing solution to the corresponding algebraic Riccati equation of (7) so far. Although we will introduce the formal assumptions shortly, let us assume that such a solution exists and $\Sigma$ satisfies the constraint arising due to this.

Now, let us make the following assumptions:

Assumption 3.1. Assume that $\hat{x}_{t}$ exists for all $t \in[0, \infty)$ and that

$$
\begin{aligned}
& \limsup _{t \rightarrow \infty} \frac{1}{t} \log E\left[\operatorname { e x p } \mu \left\{\int_{0}^{t} l\left(x_{s}, \hat{x}_{s}\right) \mathrm{d} s\right.\right. \\
& \left.\left.\quad+\tilde{l}\left(x_{t}, \hat{x}_{t}\right)\right\} \mid \mathscr{Y}_{t}\right]=c_{1}<\infty \text { for some } c_{1}>0 .
\end{aligned}
$$

Remark 3.4. In order that this assumption is satisfied, a necessary condition is that $\mu$ be sufficiently small. In general, it is very hard to derive an upper bound on $\mu$, except for some special cases (e.g. the scalar version of (1)). Here, without making matters any more complicated, we assume $\mu$ to be sufficiently small, such that (10) holds.

Assumption 3.2. There exists a bounded symmetric matrix function $\Sigma_{t} \geqslant 0$ for $t \in[0, \infty)$ that is absolutely continuous, differentiable a.e., and satisfies (9) such that the unforced linear time-varying system

$\dot{\Phi}_{t}=\left[F-\Sigma_{t}\left(H^{*} H-\mu I\right)\right] \Phi_{t}, \quad \Phi_{0}=I$

is exponentially stable.

Remark 3.5. Note that the statement of this assumption is taken following [20]. It can be also shown following [20] that Assumption 3.2 implies the following:

There exists a symmetric matrix $Q \geqslant 0$ that satisfies the algebraic Riccati equation

$$
F Q+Q F^{*}-Q\left(H^{*} H-\mu I\right) Q+\Sigma_{w}=0
$$

and $F-Q\left(H^{*} H-\mu I\right)$ is stable (i.e., has all eigenvalues in the open left half plane), such that $\lim _{t \rightarrow \infty} Q_{t}$ exists and equals $Q$.

It has been implicitly assumed above (in (7)) that $0 \leqslant \Sigma \leqslant Q$.

Remark 3.6. It has been shown in [20] that the solutions of (7) and (9) asymptotically approach $Q$, where $Q$ is defined in Assumption 3.2. 
Remark 3.7. Define the set of eigenvalues of $(F-$ $\left.Q\left(H^{*} H-\mu I\right)\right)$ to be $\overline{\mathscr{L}}$ and $\bar{\lambda}_{1}=\min \{-\operatorname{Re} \lambda \mid \lambda \in$ $\overline{\mathscr{L}}\}$. Following a similar result in [21], it follows from (11) that for any $0<\sigma_{1}<\bar{\lambda}_{1}$, there exists a constant $M_{2}$ such that

$\left\|\Phi_{t}\right\| \leqslant M_{2} \exp \left(-\sigma_{1} t\right), \quad \forall t \geqslant 0$.

Definition 3.2. Define $\mathscr{L}$ to be the set of the eigenvalues of $F-Q H^{*} H$. Define

$\bar{\lambda}=\min \{-\operatorname{Re} \lambda \mid \lambda \in \mathscr{L}\}$.

The following facts are the consequences of Assumption 3.2.

1. For every $0<\sigma<\bar{\lambda}$, there exists an $M_{\sigma}$ such that, if $\Psi_{t}$ is the transition matrix associated with $(F-$ $\left.\Sigma_{t} H^{*} H\right)$, then

$$
\left\|\Psi_{t} \Psi_{s}^{-1}\right\| \leqslant M_{\sigma} \exp (-\sigma(t-s)) \quad \text { for } t>s \geqslant 0,
$$

2.

$$
\left\|Q_{t}-\Sigma_{t}\right\| \leqslant M_{\sigma} \exp (-\sigma t), \quad \forall t \geqslant 0 .
$$

Note that the first consequence above is really a result on the exponential stability of the transition matrix associated with $\left(F-\Sigma_{t} H^{*} H\right)$. For an outline of the proof for an analogous result, see [21] where a theorem on uniform asymptotic stability has been used from [16]. Similar conditions hold in our case and hence, we do not detail the proof. The second consequence naturally follows from the fact that the solutions of (7) and (9) approach the steady state solution $Q$ exponentially fast. Again, analogous statements can be found in [21]. Here, we assume that $\sigma \neq \sigma_{1}$.

Definition 3.3. Define

$$
\begin{aligned}
\mathrm{d} v_{t} & =\mathrm{d} y_{t}-H \hat{x}_{t}^{G} \mathrm{~d} t \\
& =H\left(x_{t}-\hat{x}_{t}^{G}\right) \mathrm{d} t+\mathrm{d} v_{t} .
\end{aligned}
$$

Note that unlike the case of minimum variance filtering (or Kalman filtering), $v_{t}$ (which is known as the "innovations process" in the case of Kalman filtering) is not a Wiener process under $P$ (or $\bar{P}$ ). However, writing $\left(x_{t}-\hat{x}_{t}^{G}\right)=\tilde{e}_{t}$, one can derive the following stochastic differential equation obeyed by $\tilde{e}_{t}$, using (1) and (6):

$\mathrm{d} \tilde{e}_{t}=\left(F-Q_{t} H^{*} H\right) \tilde{e}_{t} \mathrm{~d} t+G \mathrm{~d} w_{t}-Q_{t} H^{*} \mathrm{~d} v_{t}$,

$\tilde{e}_{0}=x_{0}$.
Defining $\tilde{\Psi}_{t}$ as the transition matrix associated with $\left(F-Q_{t} H^{*} H\right)$ where $\tilde{\Psi}_{0}=I$, one can prove similar stability properties like (15) for $\tilde{\Psi}_{t}$ from the stability of the RDE (7). Using this fact and also the fact that $w_{t}$ and $v_{t}$ are mutually independent Brownian motions under $P$, and $x_{0}$ is (which is independent of $w_{t}$ and $v_{t}$ ) distributed with a Gaussian distribution having finite second-order moment, it is then straightforward to prove the following lemma.

Lemma 3.1.

$E\left|x_{t}-\hat{x}_{t}^{G}\right|^{2} \leqslant K_{e}<\infty, \quad \forall t \in[0, \infty)$

where $K_{e}$ is a constant independent of $t$.

With the above results holding, we can now present the main theorem of this section (the proof of which is not provided since it is a minor variation of a similar proof in [21] for Theorem 2.3).

Theorem 3.2. Suppose $x_{0} \sim \mathrm{N}(0, \Sigma)$. Consider (6)(9). Also suppose that Assumptions 3.1 and 3.2 hold. Then

$\lim _{t \rightarrow \infty} E\left|\hat{x}_{t}^{G}-\beta_{t}\right|^{2}=0$

Remark 3.8. Note that the zero mean assumption for the initial density is not restrictive, since with any other value for the mean and an arbitrary value for $\beta_{0}$ (other than zero) one can show that the difference between these two values is weighted by an exponentially decaying matrix and approaches 0 asymptotically. Similar results can be found in [21].

Remark 3.9. One can show in an analogous manner, that $\beta_{t}$ asymptotically converges in the mean square sense to $\beta_{t}^{R}$ where $\beta_{t}^{R}$ is given by the following equations:

$\mathrm{d} \beta_{t}^{R}=\left(F-\Sigma_{t}^{R} H^{*} H\right) \beta_{t}^{R} \mathrm{~d} t+\sum_{t}^{R} H \mathrm{~d} y_{t}, \quad \beta_{0}^{R}=0$,

where $\Sigma_{t}^{R}$ satisfies the Riccati differential equation

$$
\begin{aligned}
& \dot{\Sigma}_{t}^{R}=F \Sigma_{t}^{R}+\Sigma_{t}^{R} F^{*}+\Sigma_{w}-\Sigma_{t}^{R}\left(H^{*} H-\mu I\right) \Sigma_{t}^{R}, \\
& \Sigma_{0}^{R}=R .
\end{aligned}
$$

Here implicitly, we assume that $R<Q$. In other words, one can show that the optimal risk-sensitive estimate asymptotically converges in the mean square sense to a suboptimal risk-sensitive estimate with any arbitrary Gaussian initial condition assumption. 


\section{Risk-sensitive estimation for non-Gaussian initial conditions}

In this section, we present the risk-sensitive estimation results for non-Gaussian initial conditions. We derive an expression for the optimal risk-sensitive estimate which is finite-dimensional and a sum of two terms, where the second term shows an explicit dependence on the initial condition, and the first term, unlike the case of conditional mean estimation [21,18], also depends on the parameters of the distribution of the initial state vector. However, it is shown that under some reasonable conditions, the second term decays exponentially and the first term approaches the corresponding term for Gaussian initial conditions. These results are derived following the derivation in $[4$, Section 6.1].

Consider the signal model (1). Let $x_{0}$ have a density function $\Pi_{0}\left(x_{0}\right)$ which is non-Gaussian. We assume without loss of generality [23], that $\Pi_{0}$ has zero mean. An argument similar to Remark 3.8 can be used to justify this. Other assumptions on $\Pi_{0}$ will be introduced later. Consider also the risk-sensitive cost given by (5) and (3). Below, we define the risk-sensitive information state in a slightly modified way than it has been defined in [10] or equivalently in discrete-time, in [13].

Definition 4.1. Define the unnormalized conditional measure $q_{t}(x, \xi)$ where

$$
\begin{aligned}
& q_{t}(x, \xi) \mathrm{d} x=\bar{E}\left[\Lambda_{t} \exp \left(\mu \int_{0}^{t} l\left(x_{s}, \hat{x}_{s}\right) \mathrm{d} s\right)\right. \\
& \left.\quad \times I\left(x_{t} \in \mathrm{d} x\right) \mid \mathscr{Y}_{t}, x_{0}=\xi\right], \quad q_{0}(x, \xi)=\delta(x-\xi) .
\end{aligned}
$$

Remark 4.1. Note that, we have defined the unnormalized measure above conditioned on the initial state $x_{0}=\xi$ and hence it is function of $\xi$. It can also be interpreted as the fundamental solution of the modified Zakai equation (given below) satisfied by the risk-sensitive information state $q_{t}(x)$ defined in $[9,11]$. They are related by the following:

$q_{t}(x)=\int_{\mathbb{R}^{n}} q_{t}(x, \xi) \Pi_{0}(\xi) \mathrm{d} \xi$.

It trivially follows from the above definition that the optimal risk-sensitive estimate is given by

$\hat{x}_{t} \in \underset{\zeta}{\operatorname{argmin}} \int_{\mathbb{R}^{n}} \int_{\mathbb{R}^{n}} q_{t}(x, \xi) \exp (\mu \tilde{l}(x, \zeta)) \Pi_{0}(\xi) \mathrm{d} x \mathrm{~d} \xi$.
It is also obvious from the above Definition 4.1 that the information state achieves an expression similar to that for the information state with known initial state vector. It is also known from results on risk-sensitive information states $[5,10]$ that for a linear Gaussian systems with known initial state vector, the risk-sensitive information state achieves a Gaussian form. The proof usually follows from the fact that the Gaussian expression satisfies a modified Zakai equation (given below) and that the solution is unique provided some regularity conditions are imposed on $\Pi_{0}(\xi)$ (for similar proofs in the minimum variance case, see [4]).

The fundamental solution of the risk-sensitive information state satisfies the following stochastic partial differential equation [11] which is a modified version of the Zakai equation

$$
\begin{aligned}
\mathrm{d} q_{t}(x, \xi)= & \left(\frac{1}{2} \operatorname{Tr}\left(D^{2} q_{t}(x, \xi) \Sigma_{w}\right)-F q_{t}(x, \xi)\right. \\
& -(F x)^{*} D q_{t}(x, \xi)+\mu l\left(x, \hat{x}_{t}\right) q_{t}(x, \xi) \mathrm{d} t \\
& +q_{t}(x, \xi)\left(H_{t} x\right)^{*} \mathrm{~d} y_{t}, \quad q_{0}(x, \xi)=\delta(x-\xi) .
\end{aligned}
$$

The proof can be found in [4] or [5].

We now state the following lemma which gives the solution to the above equation.

Lemma 4.1. The risk-sensitive information state defined as the unnormalized conditional measure in Definition 4.1 for the linear time-invariant system (1) is given by

$$
\begin{aligned}
& q_{t}(x, \xi)=s_{t}(\xi) \exp \left(-\frac{1}{2}\left(x-m_{t}(\xi)\right)^{*} \Sigma_{t}^{-1}\left(x-m_{t}(\xi)\right)\right), \\
& q_{0}(x, \xi)=\delta(x-\xi),
\end{aligned}
$$

where $\Sigma_{t}$ satisfies the Riccati differential equation (9), $m_{t}(\xi)$ is given by the following equations:

$$
\begin{aligned}
m_{t}(\xi)= & \beta_{t}^{\mathrm{NG}}+\Phi_{t} \xi, \quad m_{0}(\xi)=\xi, \\
\mathrm{d} \beta_{t}^{\mathrm{NG}}= & \left(F+\mu \Sigma_{t}\right) \beta_{t}^{\mathrm{NG}} \mathrm{d} t-\mu \Sigma_{t} \hat{x}_{t}^{\mathrm{NG}} \mathrm{d} t \\
& +\Sigma_{t} H^{*}\left(\mathrm{~d} y_{t}-H \beta_{t}^{\mathrm{NG}} \mathrm{d} t\right), \quad \beta_{0}^{\mathrm{NG}}=0,
\end{aligned}
$$

where $\Phi_{t}$ is given by (11). Also, $s_{t}(\xi)$ (ignoring the terms that do not depend on $\xi$ or do not contain a random differential term since they do not affect the results due to a subsequent normalization) is given by

$s_{t}(\xi)=\gamma_{t} \exp \left[-\frac{1}{2} \xi^{*} L_{t} \xi+\xi^{*} \rho_{t}^{\mathrm{NG}}\right]$,

$\gamma_{t}=\exp \left(\int_{0}^{t}\left(H \beta_{s}^{\mathrm{NG}}\right)^{*} \mathrm{~d} y_{s}-\frac{1}{2} \int_{0}^{t}\left(H \beta_{s}^{\mathrm{NG}}\right)^{*}\left(H \beta_{s}^{\mathrm{NG}}\right)\right)$, 


$$
\begin{aligned}
& \gamma_{0}=1, \\
& L_{t}=\int_{0}^{t} \Phi_{s}^{*}\left(H^{*} H-\mu I\right) \Phi_{s} \mathrm{~d} t, \quad L_{0}=0 \\
& \mathrm{~d} \rho_{t}^{\mathrm{NG}}=\Phi_{t}^{*}\left(H^{*} \mathrm{~d} y_{t}-H^{*} H \beta_{t}^{\mathrm{NG}} \mathrm{d} t\right. \\
& \left.\quad+\mu\left(\beta_{t}^{\mathrm{NG}}-\hat{x}_{t}^{\mathrm{NG}}\right) \mathrm{d} t\right), \quad \rho_{0}^{\mathrm{NG}}=0 .
\end{aligned}
$$

Proof. First of all, the Gaussian structure of the information state given by (27) follows by showing that it satisfies the modified Zakai equation (26). Following the approach in [4], it can be shown that the solution is unique.

In view of the fact we are considering a linear Gaussian system with exponential of quadratic cost, the mean of the information state (as a function of $\xi)$ naturally assumes an affine structure like that given in (28) (this approach is similar to that in [4]). $\beta_{t}^{\mathrm{NG}}, \rho_{t}^{\mathrm{NG}}, \hat{x}_{t}^{\mathrm{NG}}$ bear the superscript NG to denote that we are dealing with non-Gaussian initial conditions.

From here onwards, much of the proof is application of Ito's rule and tedious algebra. We outline the proof below.

One can start with an unnormalized Gaussian structure like (27) for $q_{t}(x, \xi)$ and substitute in (26) to obtain the RDE for $\Sigma_{t}$, and the SDE for $m_{t}(\xi)$. Alternatively, one can verify that (27) along with (28), (9), (11) and (29), satisfy (26).

The expression for $s_{t}(\xi)$ as given by (29) is derived following a similar approach in [4]. Here, we apply Ito's rule and collect terms that are linear or quadratic functions of $\xi$ or contain random differential terms. The rest of the terms are ignored because they do not contribute to a final solution due to a subsequent normalization.

The details of the proof involve tedious algebra and not given here to maintain the readability of the paper.

Remark 4.2. Note that the above expression for $q_{t}(x, \xi)$ is an unnormalized Gaussian density. Note also that the normalization constant and the mean are functions of $\xi$.

Before presenting the result for the optimal risk-sensitive state estimate for non-Gaussian initial conditions, let us introduce the following notations.

\section{Notation 4.1.}

$M_{t}=L_{t}-\mu \Phi_{t}^{*}\left(I-\mu \Sigma_{t}\right)^{-1} \Phi_{t}$,

$$
\begin{aligned}
& D_{t}(N)=\frac{\int_{\mathbb{R}^{n}} \xi \exp \left[-\frac{1}{2} \xi^{*} M_{t} \xi+\xi^{*} N\right] \Pi_{0}(\xi) \mathrm{d} \xi}{\int_{\mathbb{R}^{n}} \exp \left[-\frac{1}{2} \xi^{*} M_{t} \xi+\xi^{*} N\right] \Pi_{0}(\xi) \mathrm{d} \xi}, \\
& N_{t}^{\mathrm{NG}}=\rho_{t}^{\mathrm{NG}}-\mu \Phi_{t}^{*}\left(I-\mu \Sigma_{t}\right)^{-1} \Phi_{t} D_{t}\left(N_{t}^{\mathrm{NG}}\right) .
\end{aligned}
$$

Remark 4.3. Note the following things about the above notations. It has been assumed that $\mu$ is small enough such that $\left(I-\mu \Sigma_{t}\right)>0 . N_{t}^{\mathrm{NG}}$ is a stochastic process and is defined in an implicit fashion. The existence of $N_{t}^{\mathrm{NG}}$ follows (in the sense that a solution to the above implicit equation in Notation 4.1 exists) from its relation with $\hat{x}_{t}^{\mathrm{NG}}$ given in (32) and from the assumed existence of $\beta_{t}^{\mathrm{NG}}$ and $\rho_{t}^{\mathrm{NG}}$ as solutions to the stochastic differential equations given in Lemma 4.1 and that of $\hat{x}_{t}^{\mathrm{NG}}$ as in Assumption 3.1.

We will introduce formal assumptions on $\Pi_{0}($.$) be-$ low, but for the time being we assume that the denominator in $D_{t}\left(N_{t}^{\mathrm{NG}}\right)$ is well-defined. Also, note that $M_{t}$ might not be positive-definite $\forall t$.

It is obvious that $N_{t}^{\mathrm{NG}}$ is a $\left\{\mathscr{Y}_{t}\right\}$-adapted process and $\bar{\Lambda}_{t}=\exp \left[-(1 / 2) x_{0}^{*} M_{t} x_{0}+x_{0}^{*} N_{t}^{\mathrm{NG}}\right] / \int_{\mathbb{R}^{n}} \exp [-(1 / 2)$ $\left.\xi^{*} M_{t} \xi+\xi^{*} N_{t}^{\mathrm{NG}}\right] \Pi_{0}(\xi) \mathrm{d} \xi$ is $\left\{\mathscr{Y}_{t} \vee \sigma\left\{x_{0}\right\}\right\}$-adapted. Using this notation, we can re-write $D_{t}\left(N_{t}^{\mathrm{NG}}\right)$ as

$D_{t}\left(N_{t}^{\mathrm{NG}}\right)=E_{x_{0}}\left[x_{0} \bar{\Lambda}_{t} \mid \mathscr{Y}_{t}\right]$,

where $E_{x_{0}}$ denotes expectation with respect to $\Pi_{0}\left(x_{0}\right)$. Note also that $E_{x_{0}}\left[\bar{\Lambda}_{t}\right]=1, \forall t$.

In other words, one can think of $D_{t}\left(N_{t}^{\mathrm{NG}}\right)$ as the conditional expectation of $x_{0}$ under a different probability measure $\tilde{P}$ such that $\mathrm{d} \tilde{P} / \mathrm{d} P=\bar{\Lambda}_{t}$. Now, we make the following assumptions that lead to the well-defined property and boundedness of $D_{t}\left(N_{t}^{\mathrm{NG}}\right)$ in the mean square sense.

Assumption 4.1. $\mu$ is chosen small enough such that $H^{*} H-\mu I>0$.

Remark 4.4. Note that this restriction is not necessary but in conjunction with the following assumptions, sufficient to prove the asymptotic optimality of the risk-sensitive estimates for non-Gaussian initial conditions. Also, $\mu$ is a design parameter and the well-defined property of the risk-sensitive filter heavily depends on $\mu$ being small enough. Note that the theory of Section 3 does not change in view of the above assumption although Assumption 3.2 becomes unnecessary since from the theory of Kalman filtering Riccati equations, it becomes a fact.

The other implication of this assumption is that on the behaviour of $L_{t}$ and $M_{t}$. In the scalar case, it is easy to notice the following. Obviously, from (29), it 
is clear that $L_{t} \geqslant 0, \forall t$ with $L_{0}=0$ and $L_{\infty}<\infty$, where $L_{\infty}$ is the steady-state solution for the equation for $L_{t}$ in (29). Now, if we look at the equation for $M_{t}$ in (30), it is easy to deduce that $M_{0}=-\mu$ and $M_{\infty}=L_{\infty}$. Obviously, in some intermediate time period, $M_{t}<0$. Although, we are only investigating solutions as $t \rightarrow$ $\infty$, we need to make sure that the risk-sensitive filter does not become unstable for some time $t<\infty$. We now make the following formal assumptions.

Assumption 4.2. There exists a $0<M_{x}<\infty$ such that $E\left[\left|x_{0}\right|^{4}\right]<M_{x}$.

Assumption 4.3. $\Pi_{0}($.$) has such boundedness proper-$ ties that $\bar{\Lambda}_{t}$ is well-defined for $M_{t}<0$.

Assumption 4.4. There exists a $M_{d}>0$ and $0<\sigma_{d}$ $<\sigma_{1}$ for some $0<\sigma_{1}<\bar{\lambda}_{1}$ such that $\bar{\Lambda}_{t}$ is a $\left\{\mathscr{Y}_{t} \vee \sigma\left\{x_{0}\right\}\right\}$-adapted process where $E\left[\bar{\Lambda}_{t}^{4}\right] \leqslant M_{d}$ $\exp \left(4 \sigma_{d} t\right)$.

Remark 4.5. Note that Assumptions 4.2 and 4.4 together imply that $E\left|D_{t}\left(N_{t}^{\mathrm{NG}}\right)\right|^{2} \leqslant M_{N} \exp \left(2 \sigma_{d} t\right)$, $\forall t \in[0, \infty)$. To see this, note that $\left|D_{t}\left(N_{t}^{\mathrm{NG}}\right)\right|^{2} \leqslant$ $E_{x_{0}}\left[\left|x_{0}\right|^{2} \bar{\Lambda}_{t}^{2} \mid \mathscr{Y}_{t}\right]$ from Jensen's inequality. Hence $E\left|D_{t}\left(N_{t}^{\mathrm{NG}}\right)\right|^{2} \leqslant E\left[\left|x_{0}\right|^{2} \bar{\Lambda}_{t}^{2}\right] \leqslant \sqrt{E\left[\left|x_{0}\right|^{4}\right]} \sqrt{E\left[\bar{\Lambda}_{t}^{4}\right]}$ where the last step follows from Schwartz's inequality. Now, using Assumptions 4.2 and 4.4, it follows that $E\left|D_{t}\left(N_{t}^{\mathrm{NG}}\right)\right|^{2} \leqslant M_{N} \exp \left(2 \sigma_{d} t\right), \forall t \in[0, \infty)$ where $M_{N}>0$ is a constant. One can possibly look for a sufficient condition by imposing regularity properties on $\Pi_{0}($.$) and boundedness properties on the process$ $N_{t}^{\mathrm{NG}}$ such that Assumption 4.4 is satisfied. But due to the complicated nature of the process $N_{t}^{\mathrm{NG}}$ we postpone such investigation for the time being. However, since this assumption on the growth of $E\left|D_{t}\left(N_{t}^{\mathrm{NG}}\right)\right|^{2}$ is still allowed to be exponential with a slow enough growth, we consider it to be not too restrictive.

With the above definitions and assumptions holding, we present the following theorem.

Theorem 4.1. Consider the signal model (1) with $x_{0} \sim \Pi_{0}\left(x_{0}\right)$. Consider also the risk-sensitive estimation problem defined by (2) and (3). Then, the risk-sensitive estimate for non-Gaussian initial conditions $\hat{x}_{t}^{\mathrm{NG}}$ is given by the following equation:

$\hat{x}_{t}^{\mathrm{NG}}=\beta_{t}^{\mathrm{NG}}+\Phi_{t} D_{t}\left(N_{t}^{\mathrm{NG}}\right), \quad \hat{x}_{0}^{\mathrm{NG}}=0$.
Proof. The proof is straightforward once we note that we can substitute the expression for $q_{t}(x, \xi)$ from (27) with (28) and (29) holding, in (25). Completion of squares and integrating over the Gaussian density with respect to $x$ leave us with the integration over $\xi$ which we cannot perform since we do not have an expression for $\Pi_{0}(\xi)$. From the nature of the convex cost that goes to infinity as $\left|\hat{x}_{t}^{\mathrm{NG}}\right|$ goes to infinity, it is obvious that there exists a unique infimum which can be obtained by putting the derivative of the expression on the right-hand side of (25) to zero. The rest of the proof is just algebra and not shown here.

Remark 4.6. It is well known that as $\mu \rightarrow 0$, one recovers the minimum variance estimation problem. One can verify that by substituting $\mu=0$ in the above equations, one can obtain the results for conditional mean estimation for linear Gaussian systems with non-Gaussian initial conditions as given in [18].

It is also noteworthy that the optimal risk-sensitive estimate is expressed in terms of a finite number of statistics $\beta_{t}^{\mathrm{NG}}, N_{t}^{\mathrm{NG}}, \Sigma_{t}, \Phi_{t}$ and $M_{t}$. The difference with the minimum variance case (the results of [18]) lies in the fact that $N_{t}^{\mathrm{NG}}$ is given by an implicit equation (30) which makes the computation more difficult. However, one can easily see that putting $\mu=0$ would recover the minimum variance estimation results (as mentioned above) and such problems with implicit equations will disappear. Also, in the case of Gaussian initial conditions, one can obtain an explicit solution for $D_{t}\left(N_{t}^{\mathrm{NG}}\right)$ which can be substituted back in (32) to obtain the SDE (6).

\subsection{Asymptotic optimality of risk-sensitive filters for non-Gaussian initial conditions}

In this section, we present the results on the mean square asymptotic convergence of the optimal risk-sensitive estimate to the suboptimal risk-sensitive estimate with a Gaussian initial condition assumption with zero mean and zero covariance (defined as $\beta_{t}$ in Section 3, (8)).

The following theorem summarizes the result of this section.

Theorem 4.2. Consider the signal model (1) where $x_{0} \sim \Pi_{0}\left(x_{0}\right), \Pi_{0}$ being non-Gaussian. Consider also the risk-sensitive estimation problem given by (2) and (3). Suppose Assumptions 4.3 and 4.4 hold. Then the optimal risk-sensitive estimate given by (32) 
asymptotically converges to the suboptimal risksensitive estimate given by (8) and (9) in the mean square sense, that is,

$\lim _{t \rightarrow \infty} E\left|x_{t}^{\mathrm{NG}}-\beta_{t}\right|^{2} \rightarrow 0$.

Proof. Note that from Remark 4.5, we have $E\left|D_{t}\left(N_{t}^{\mathrm{NG}}\right)\right|^{2} \leqslant M_{N} \exp \left(2 \sigma_{d} t\right), \forall t \in[0, \infty)$.

Using Minkowski's inequality, one can write

$E\left|\hat{x}_{t}^{\mathrm{NG}}-\beta_{t}\right|^{2} \leqslant\left\{\sqrt{E\left|\hat{x}_{t}^{\mathrm{NG}}-\beta_{t}^{\mathrm{NG}}\right|^{2}}+\sqrt{E\left|\beta_{t}^{\mathrm{NG}}-\beta_{t}\right|^{2}}\right\}^{2}$.

Note from (28) and (32) that one can write

$\mathrm{d} \beta_{t}^{\mathrm{NG}}=\left(F-\Sigma_{t} H^{*} H\right) \beta_{t}^{\mathrm{NG}} \mathrm{d} t-\mu \Sigma_{t} \Phi_{t} D_{t}\left(N_{t}^{\mathrm{NG}}\right) \mathrm{d} t$

$$
+\Sigma_{t} H^{*} \mathrm{~d} y_{t} \text {. }
$$

Comparing this with (8), we have

$$
\begin{aligned}
d\left(\beta_{t}^{\mathrm{NG}}-\beta_{t}\right)= & \left(F-\Sigma_{t} H^{*} H\right)\left(\beta_{t}^{\mathrm{NG}}-\beta_{t}\right) \mathrm{d} t \\
& -\mu \Sigma_{t} \Phi_{t} D_{t}\left(N_{t}^{\mathrm{NG}}\right) \mathrm{d} t
\end{aligned}
$$

which immediately gives us

$$
\begin{aligned}
& E\left|\beta_{t}^{\mathrm{NG}}-\beta_{t}\right|^{2} \\
& \leqslant \mu^{2} E\left|\int_{0}^{t} \Psi_{t} \Psi_{s}^{-1} \Sigma_{s} \Phi_{s} D_{s}\left(N_{s}^{\mathrm{NG}}\right) \mathrm{d} s\right|^{2} \\
& \leqslant \mu^{2} E\left|\int_{0}^{t}\left\|\Psi_{t} \Psi_{s}^{-1}\right\|\left\|\Sigma_{s}\right\|\left\|\Phi_{s}\right\|\right| D_{s}\left(N_{s}^{\mathrm{NG}}\right)|\mathrm{d} s|^{2} \\
& \leqslant \mu^{2} M_{\sigma, \sigma_{e}} t \exp \left(-2 \sigma_{e} t\right)\left[1-\exp \left[-2\left(\sigma-\sigma_{e}\right) t\right]\right] \\
& \leqslant \mu^{2} M_{\sigma, \sigma_{e}} t \exp \left(-2 \sigma_{e} t\right),
\end{aligned}
$$

where $0<\sigma_{e}=\sigma_{1}-\sigma_{d}$ and we have used (15), (13), the fact that $\Sigma_{t}$ is bounded (from Assumption 3.2) $\forall t$, and Jensen's inequality. $M_{\sigma, \sigma_{e}}>0$ is a constant independent of $t$. We have also arbitrarily taken $\sigma-$ $\sigma_{e}=\bar{\gamma}>0$. If $\bar{\gamma}<0$, one could obtain the following expression for the above bound:

$E\left|\beta_{t}^{\mathrm{NG}}-\beta_{t}\right|^{2} \leqslant \mu^{2} \tilde{M}_{\sigma, \sigma_{e}} t \exp (-2 \sigma t)$,

where $\tilde{M}_{\sigma, \sigma_{e}}>0$ is a constant independent of $t$. Note that $\sigma, \sigma_{1}$ can be chosen such that $\bar{\gamma} \neq 0$.

Also, from (13), we have

$E\left|\hat{x}_{t}^{\mathrm{NG}}-\beta_{t}^{\mathrm{NG}}\right|^{2} \leqslant M_{2}^{2} \exp \left(-2 \sigma_{e} t\right)$.

Going back to (34), it is obvious from (37)-(39) that $t \rightarrow \infty$, we have

$\lim _{t \rightarrow \infty} E\left|\hat{x}_{t}^{\mathrm{NG}}-\beta_{t}\right|^{2} \rightarrow 0$.

Corollary 4.1. Consider the signal model (1) where $x_{0} \sim \Pi_{0}\left(x_{0}\right), \Pi_{0}$ being non-Gaussian. Consider also the risk-sensitive estimation problem given by (2) and (3). Suppose Assumptions 4.3 and 4.4 hold. Then the optimal risk-sensitive estimate given by (32) asymptotically converges to the suboptimal risk-sensitive estimate given by (21) and (22) in the mean square sense, that is,

$$
\lim _{t \rightarrow \infty} E\left|x_{t}^{\mathrm{NG}}-\beta_{t}^{R}\right|^{2} \rightarrow 0 .
$$

Proof. It follows easily from Remark 3.9.

\section{Conclusion}

In this paper, we have addressed the problem of risk-sensitive estimation with non-Gaussian initial conditions. We present the risk-sensitive filtering results for linear Gaussian systems with Gaussian initial conditions and show that a suboptimal risk-sensitive filter with zero initial covariance (the extension to any arbitrary initial covariance is possible as long as it results in a stabilizing solution of the corresponding algebraic Riccati equation) asymptotically approaches the optimal risk-sensitive filter with the true initial covariance in the mean square sense. Next, we derive the expressions for a conditional measure which we call as the risk-sensitive information state and those for the optimal risk-sensitive estimate for non-Gaussian initial conditions. We show that the optimal risk-sensitive estimate can be expressed in terms of a finite number of statistics. Lastly, we show that the suboptimal risk-sensitive estimate with zero initial covariance (or any arbitrary Gaussian initial conditions that satisfy the constraint mentioned above) asymptotically approaches the optimal risk-sensitive estimate with non-Gaussian initial conditions in the mean square sense under certain conditions. Finally, one can recover the corresponding conditional mean (or minimum variance) estimation results for non-Gaussian initial conditions by letting $\mu \rightarrow 0$.

\section{Acknowledgements}

The authors are very grateful to Dr. Matthew James and Prof. Robert J. Elliott and Professor Andre Tits for many helpful suggestions and insights.

\section{References}

[1] R. Atar, O. Zeitouni, Exponential stability for nonlinear filtering, preprint, 1996. 
[2] R. Atar, O. Zeitouni, Lyapunov exponents for finite-state nonlinear filtering, SIAM J. Control \& Optimization 35(1) (1997) 36-55.

[3] V.E. Benes, Exact finite-dimensional filters for certain diffusions with nonlinear drift, Stochastics 5 (1981) 65-92.

[4] A. Bensoussan, Stochastic Control of Partially Observable Systems, Cambridge University Press, Cambridge, UK, 1992.

[5] A. Bensoussan, R.J. Elliott, General finite dimensional risk sensitive problems and small noise limits, IEEE Trans. Automat. Control 41(2) (1996) 210-215.

[6] R.K. Boel, M.R. James, I.R. Petersen, Robust filtering, Proceedings of the Conference on Decision and Control, San Diego, December 1997.

[7] A. Budhiraja, D. Ocone, Exponential stability of discrete-time filters for bounded observation noise, System Control Lett. 30 (1997) 185-194.

[8] C.D. Charalambous, The role of information state and adjoint in relating nonlinear output feedback risk-sensitive control and dynamic games, IEEE Trans. Automat. Control 42(8) (1997) 1163-1170.

[9] C.D. Charalambous, S. Dey, R.J. Elliott, New finitedimensional risk-sensitive filters: Small-noise limits, IEEE Trans. Automat. Control 43(10) (1997) 1424-1429.

[10] C.D. Charalambous, D.S. Naidu, K.L. Moore, Solvable risksensitive control problems with output feedback, Proceedings of the 33rd IEEE Conference on Decision and Control, Lake Buena Vista, Florida, December, 1994, pp. 1433-1434.

[11] S. Dey, R.J. Elliott, J.B. Moore, Finite-dimensional risk-sensitive estimation for continuous-time nonlinear systems, Proceedings of the European Control Conference, Brussels, July 1997.

[12] S. Dey, J.B. Moore, Risk-sensitive filtering and smoothing for hidden markov models, Systems Control Lett. 25(5) (1995) 361-366.

[13] S. Dey, J.B. Moore, Risk-sensitive filtering and smoothing via reference probability methods, IEEE Trans. Automat. Control 42(11) (1997) 1587-1591.

[14] B. Deylon, O. Zeitouni, Lyapunov exponents for filtering problems, in: M.H.A. Davis, R.J. Elliott (Eds.), Applied
Stochastic Analysis, Gordon and Breach, New York, 1991, pp. 511-535.

[15] R.J. Elliott, L. Aggoun, J.B. Moore, Hidden Markov Models: Estimation and Control, Springer, Berlin, 1994.

[16] C.J. Harris, J.F. Miles, Stability of Linear Systems, Academic Press, New York, 1980.

[17] M.R. James, J.S. Baras, R.J. Elliott, Risk-sensitive control and dynamic games for partially observed discrete-time nonlinear systems, IEEE Trans. Automat. Control 39(4) (1994) 780792.

[18] A.M. Makowski, Filtering formulae for partially observed linear systems with non-gaussian initial conditions, Stochastics 16 (1986) 1-24.

[19] J.B. Moore, R.J. Elliott, S. Dey, Risk-sensitive generalizations of minimum variance estimation and control, IFAC Symposium on Nonlinear Control Systems Design, Tahoe City, CA, June 1995, pp. 465-470 (also see summary in J. Math. Systems, Estimation Control 7(1) (1997) 123-126.).

[20] K.M. Nagpal, P.P. Khargonekar, Filtering and smoothing in an $H_{\infty}$ setting, IEEE Trans. Automat. Control 36(2) (1991) $152-166$.

[21] D. Ocone, E. Pardoux, Asymptotic stability of the optimal filter with respect to its initial conditions, SIAM J. Control Optim. 34(1) (1996) 226-243.

[22] D.L. Ocone, Asymptotic stability of Benes filters, preprint.

[23] R.B. Sowers, A.M. Makowski, Discrete-time filtering for linear systems with non-gaussian initial conditions: asymptotic behaviour of the difference between the MMSE and LMSE estimates, IEEE Trans. Automat. Control 37(1) (1992) 114-120.

[24] J.L. Speyer, C. Fan, R.N. Banavar, Optimal stochastic estimation with exponential cost criteria, Proceedings of the 31st Conference on Decision and Control, Vol. 2, December 1992, pp. 2293-2298.

[25] P. Whittle, Risk-sensitive linear/quadratic/Gaussian control, Adv. Appl. Probab. 13 (1981) 764-777.

[26] P. Whittle, A risk-sensitive maximum principle: the case of imperfect state observations, IEEE Trans. Automat. Control 36(7) (1991) 793-801. 